\title{
Crystallization Process in Porous and Nonporous Vycor Glass
}

\author{
B. JAsińskA ${ }^{a}$, A.L. DAwidowicz ${ }^{b}$ And S. Pikus ${ }^{b}$ \\ ${ }^{a}$ Institute of Physics, Maria Curie-Skłodowska University \\ pl. M. Curie-Skłodowskiej 1, 20-031 Lublin, Poland \\ ${ }^{b}$ Faculty of Chemistry, Maria Curie-Skłodowska University \\ pl. M. Curie-Skłodowskiej 1, 20-031 Lublin, Poland
}

Three stages of thermal and chemical processing of Vycor glass were carried out. Raw material, the glass with small and big pores was studied. In each stage of study various crystallinity degrees of investigated material were obtained. The relation between crystallinity degree and the intensity of $o$-Ps components was determined. In nonporous material a high correlation between $o$-Ps intensity and crystallinity degree was found. In porous material the decrease in total $o$-Ps intensity can be a measure of the crystallinity degree.

PACS numbers: $78.70 . \mathrm{Bj}$

\section{Introduction}

In the last three decades, the numerous and varied applications of X-ray diffraction have established it as one of fundamental and important tools employed for characterization of crystalline of semicrystalline state. X-ray crystallography, with single crystal or powder, allows us to study the structure of crystalline samples. Besides, the X-ray diffraction, especially X-ray powder diffractometry, is very often used for qualitative and quantitative analysis, precise determination of lattice constant, measuring the crystallite size, lattice strains and stress, as well as for investigation of preferred orientation and texture. It is easy to distinguish between the X-ray diffraction pattern for crystalline and amorphous materials. In the first case, several narrow peaks are seen, while for amorphous phase only a broad distribution is present. Therefore, the X-ray diffraction methods can be used to estimate the crystallinity degree of the examined samples.

Positron annihilation lifetime spectroscopy (PALS) is often used in various material microstructure investigations. Using the model proposed by Tao [1] and 
Eldrup [2] and extended by Goworek et al. [3, 4], free volume size can be determined from the $o$-Ps lifetime, while from $o$-Ps intensity the amount of any kind of voids existing in the material can be examined. Both methods, X-ray diffraction and PALS are used in this paper to study the crystallization process in the Vycor glass containing voids ranged from a few angstroms to hundred $\mathrm{nm}$.

\section{Experimental}

Vycor glass composed of sodium, boron, and silicon oxides was used in our experiments. In the first part of our study, initial material was heated at 875, 925, 950 , and $975 \mathrm{~K}$ in periods of time changing from 0.5 to $48 \mathrm{~h}$. In the second one samples of glass were heated from 2 to $16 \mathrm{~h}$ at 925 or $950 \mathrm{~K}$ and then etched in sulphuric acid in order to obtain porous material. In such a way we could prepare material containing narrow pores of $\sim 1 \mathrm{~nm}$ radius. In the third part of the study we used porous glass (pore radii 10-100 nm) heated second time at 985, 1000, $1015 \mathrm{~K}$ from 8 to $168 \mathrm{~h}$.

$\mathrm{X}$-ray measurements were performed on DRON-3 (USRR) diffractometer using $\mathrm{Cu} K_{\alpha}$ radiation. A scanning pattern was recorded in the $2 \Theta$ range of $10-90^{\circ}$ (the scanning step equals $0.02^{\circ}$, measuring time equals $20 \mathrm{~s}$ ) for each sample. The crystallinity degree was calculated as a ratio of the integrated intensity of diffraction peaks belonging to the crystalline phase to the integrated total intensity for the sample (sum of the intensities from crystalline and amorphous phases). The values of intensity coming from crystalline and amorphous phases were estimated by Hindeleh-Johnson [5-7] method as well as its modification [8].

The PALS measurements were performed using a standard fast-slow spectrometer [9] with time resolution $0.22 \mathrm{~ns}$, at room temperature under pressure of $\sim 0.5 \mathrm{~Pa}$. The channel definition was $0.125 \mathrm{~ns}$. About $2 \times 10^{6}$ events per spectrum were collected. The time of measurements was $4 \mathrm{~h}$ per spectrum. The spectrum was deconvoluted by LT programme [10] into three components. The shortestlived component (described by $\tau_{1} \approx 0.4 \mathrm{~ns}$ ) belongs to the decay of $p$-Ps and free annihilation (not resolved because of the high channel definition), the second one ( $\tau_{2}$ from 1.2 to $1.5 \mathrm{~ns}$ ) origins from $o$-Ps decay in small intermolecular voids, while the third - the longest-lived one is produced also by the annihilation of o-Ps but in the pores. In this paper we discuss only the intensities ascribed to o-Ps components.

\section{Results and discussion}

It is seen from Fig. 1a that crystallization of annealed tree-component glass, used in this research, begins above $650^{\circ} \mathrm{C}$. From X-ray data, one can see that the crystallization degree rises with time of heating at each temperature. The increase in thermal treatment time and temperature results in the increased crystallinity 
degree. The PALS data show that in amorphous raw material one can find a component ascribed to the annihilation of $o$-Ps in small voids with the mean $o$-Ps lifetime of $\tau_{2}=1.33 \mathrm{~ns}$ and intensity $I_{2}=32 \%$. These values agree with the data known from literature $[11,12]$. The component of about $1.5 \mathrm{~ns}$ is always seen in amorphous glasses. In perfect $\mathrm{SiO}_{2}$ crystal, the intensity of this component has to be $0 \%$. From Fig. $1 \mathrm{~b}$ one can see that $o$-Ps intensity decreases with the rise in annealing temperature and time. It means that the total amount of defects in the material also decreases. Comparing the data from X-ray diffraction and PALS one can conclude that the increase in crystallinity degree is well correlated with the decrease in $o$-Ps intensity for each temperature. However, crystallinity degree never reaches $100 \%$, and $o$-Ps intensity never falls to $0 \%$. The both methods show that full crystallization in Vycor glass of this composition was not achieved. This is related to the presence of $30 \%$ of admixtures in $\mathrm{SiO}_{2}$ base. The full discussion of processes occurring in the investigated material one can find in the paper by Jasińska et al. [13].
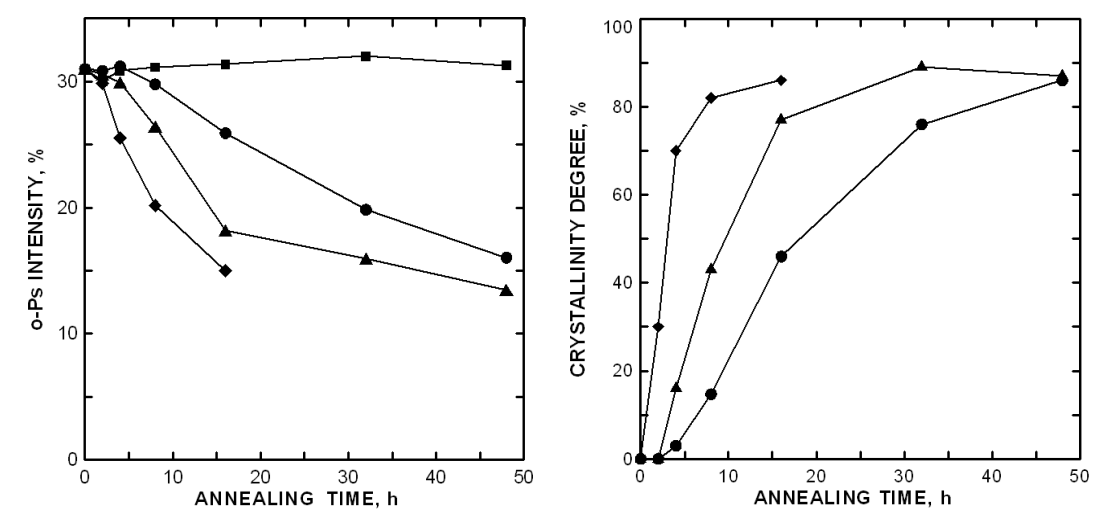

Fig. 1. The dependence of the $o$-Ps intensity (on the left) and the crystallinity degree (on the right) on the annealing time for a few selected temperatures for row glass. Points in both figures denote: squares $-875 \mathrm{~K}$, circles $-925 \mathrm{~K}$, triangles $-950 \mathrm{~K}$, diamonds — 975 K. Lines are eye-guide only.

The second stage of our experiment was carried out using porous glass after liquation and leaching [14]. In such material two o-Ps components exist: the first one ascribed to annihilation in small intermolecular voids with lifetime $\sim 1.5 \mathrm{~ns}$, and the second one connected with $o$-Ps decay in the pores. For both selected temperatures, the $o$-Ps lifetime in the pores remains almost constant and is equal to $23.5 \mathrm{~ns}$ (radius $\sim 0.9 \mathrm{~nm}$ ) at $925 \mathrm{~K}$ and $18 \mathrm{~ns}$ (radius $\sim 0.8 \mathrm{~nm}$ ) at $950 \mathrm{~K}$. It means that pore radii do not change with time of annealing at each temperature. The intensities of both $o$-Ps components change with time and temperature. $I_{2}$ is constant at lower temperature while it rapidly decreases at higher one, for heating time above $4 \mathrm{~h}$. Intensity in the pores decreases at both temperatures, however 
the rate of lowering is much faster at $950 \mathrm{~K} \mathrm{[15].} \mathrm{From} \mathrm{Fig.} 2$ it is seen that the increasing of the crystallinity degree is correlated with the decrease in total $o$-Ps intensity. The slope of curves in Fig. $2 \mathrm{a}$ and $\mathrm{b}$ for respective temperatures has the same value but the opposite sign. The same relationship can be observed in Fig. $1 \mathrm{a}$ and $\mathrm{b}$.
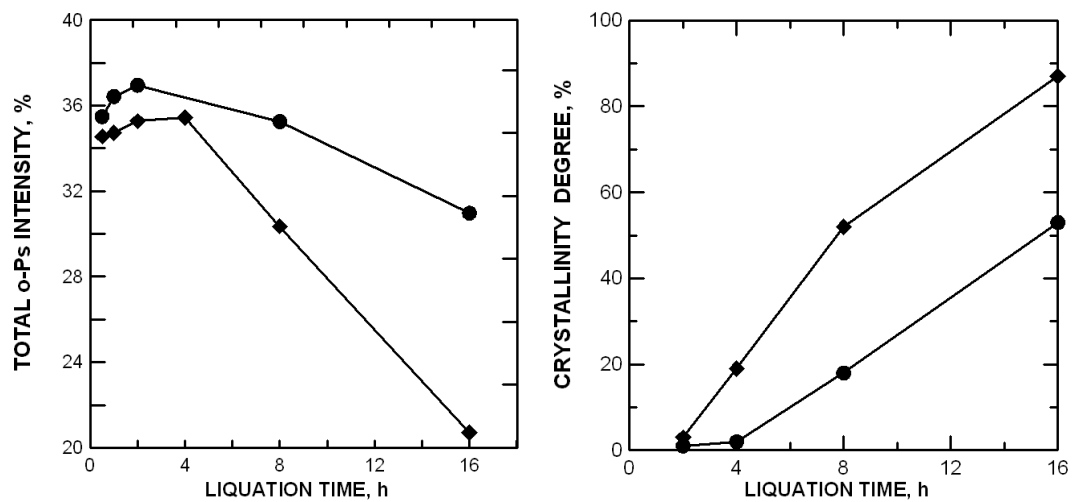

Fig. 2. Total $o$-Ps intensity (on the left) and the crystallinity degree (on the right) as a function of the annealing time for a few selected temperatures for glass with small pores. Points in both figures denotes: circles - $925 \mathrm{~K}$, diamonds - $950 \mathrm{~K}$. Lines are eye-guide only.
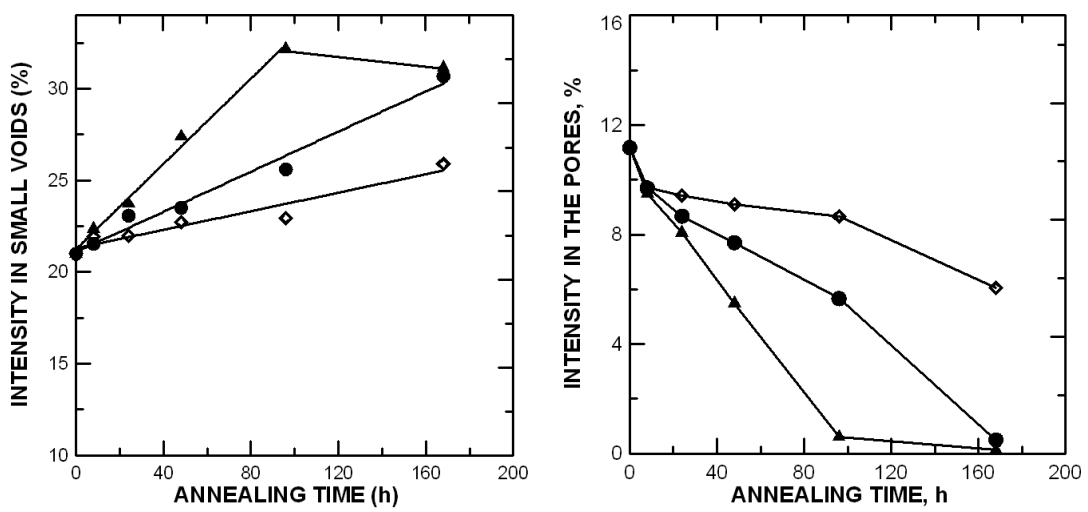

Fig. 3. The $o$-Ps intensity in small voids (on the left) and $o$-Ps intensity in the pores (on the right) vs. the annealing time for glass with big pores. Points denote: diamonds - samples annealed at $985 \mathrm{~K}$, circles — at $1000 \mathrm{~K}$ and triangles — at $1015 \mathrm{~K}$. Lines are eye-guide only.

The third stage of investigations of crystallization process in glass is now in progress. It is done with porous materials containing the pores of big diameter. The glass was annealed in order to recrystalize almost pure silica (after etching, 
admixtures were removed out of material). Figure $3 a$ and $b$ shows the changes in $o$-Ps intensity in small voids and in the pores for the selected initial sample. It was unexpected that $I_{2}$ increases with time as well as with temperature of annealing. It means that total surface of small voids increases significantly in comparison with total surface of the pores, because $I_{3}$ decreases. Total $o$-Ps intensity lowers with temperature of heating. It will be interesting to compare crystallinity degree with $o$-Ps intensity, like in both earliest stages of research.

\section{Conclusions}

Our experiments show that in studying the crystallization process PALS can be used as a complementary method to classic X-ray diffraction. Total o-Ps intensity decrease is highly correlated with the rise in the crystallinity degree in Vycor glass.

It seems that PALS can be very useful in such researches, it supplies us with several parameters like lifetimes and intensities of $o$-Ps components. From the lifetime values one can obtain the information about the sizes of various kinds of voids, from the intensities — the amount of such voids.

\section{References}

[1] S.J. Tao, J. Chem. Phys. 56, 5499 (1972).

[2] M. Eldrup, D. Lightbody, J.N. Sherwood, Chem. Phys. 63, 51 (1981).

[3] T. Goworek, K. Ciesielski, B. Jasińska, J. Wawryszczuk, Chem. Phys. Lett. 272, 91 (1997).

[4] K. Ciesielski, A.L. Dawidowicz, T. Goworek, B. Jasińska, J. Wawryszczuk, Chem. Phys. Lett. 289, 41 (1998).

[5] A.M. Hindeleh, D.J. Johnson, Polymer 13, 27 (1972).

[6] A.M. Hindeleh, D.J. Johnson, Polymer 13, 423 (1972).

[7] A.M. Hindeleh, D.J. Johnson, Polymer 15, 697 (1974).

[8] M. Rabiej, Polimery 47, 423 (2002) (in Polish).

[9] P. Hautojärvi, Positrons in Solids, Springer-Verlag, Berlin 1979.

[10] J. Kansy, Nucl. Instrum. Methods A 374, 235 (1996).

[11] C. Hugenschmidt, U. Holzwarth, M. Jansen, S. Kohn, K. Maier, J. Non-Crystalline Solids 217, 72 (1997).

[12] P. Hautojärvi, I. Lehmuoska, P. Jauho, V. Komppa, E. Pajanne, Solid State Commun. 15, 1859 (1974).

[13] B. Jasińska, A.L. Dawidowicz, S. Pikus, Phys. Chem. Chem. Phys. 5, 3289 (2003).

[14] B. Jasińska, A.L. Dawidowicz, T. Goworek, Phys. Chem. Chem. Phys. 2, 3269 (2000).

[15] B. Jasińska, A.L. Dawidowicz, S. Pikus, submitted to Phys. Chem. Chem. Phys. 